\title{
A face recognition by similarity (FRBS) conjecture
}

\author{
SAM S. RAKOVER \\ Haifa University, Haifa, Israel \\ AND \\ BARUCh CAHLON \\ Oakland University, Rochester, Michigan
}

\begin{abstract}
Cohen (1963) investigated free recall in two lists of words. The first contained unrelated words. The second comprised words drawn from several semantic categories, where the number of categories was equal to the number of words in the first list. He found that recall of unrelated words was equal to the recall of categories. The face recognition by similarity (FRBS) conjecture proposes that this relation cannot be applied to face recognition. Following Cohen's design, two different experimental situations for generating two target faces were constructed. The findings showed that the number of correct recognitions of specific facial features belonging to the first target face (e.g., nose, chin) was greater than or equal to the number of categories of visually similar facial features belonging to the second target face (e.g., different long noses, round chins). In addition, theoretical underpinnings for the FRBS conjecture were suggested.
\end{abstract}

Between the 1950s and the early 1970s, a major effort was made in memory research to discover the relation between organizational processes in verbal free recall (e.g., Baddeley, 1976; Murdock, 1974; Tulving \& Pearlstone, 1966; Wood, 1972). The general finding was that free recall was enhanced when the words in the list to be remembered were grouped under different categories.

One important question from that research program, which constituted a theoretical trigger for the present study, concerns the relationship between the recall of two different lists of words. One list, L, contains $n$ words, which are divided into $g$ categories (clusters, groups), with $z$ words per category - that is, $z=n / g$ that are semantically related. The other list, 1 , contains $n^{\prime}$ unrelated words, where $n^{\prime}=g$. For example, $\mathrm{L}$ has $n=30$ words, which are divided into $g=10$ categories with $z=3$ words per category. List 1 has $n^{\prime}=10$ unrelated words, $n^{\prime}=g$. The question is whether the number of categories that will be correctly recalled from L [denoted by $C(g, z)$, which depends on $g$ and $z$ ] will be equal to the number of words that will be correctly recalled from 1 [denoted by $W\left(n^{\prime}\right)$, which depends on $n^{\prime}$ ] - that is, whether $W\left(n^{\prime}\right)=C(g, z)$.

Cohen (1963) tested the hypothesis that $W\left(n^{\prime}\right)=C(g, z)$ (called the chunk hypothesis) under two conditions: one that fulfilled and one that did not fulfill the total-time $(T)$ requirement. Accordingly, the lists should be equal in their $T$ : the multiplication of the number of words in a list $(n)$ by the presentation time of a word $(t)$ - that is, $T=$ $n t$ - since it was found that the number of words recalled in a single-trial free recall increased as a function of $T$ (see Murdock, 1960, 1974).

On the basis of Miller's (1956) concept of a chunk (a group of words that "are related in some manner" [Cohen, 1963, p. 227]), Cohen phrased Miller's chunk hypothesis in the following manner:

A list comprising 20 categories of words and a list of 20 unrelated words are equivalent in the sense that each contains 20 units or chunks of information. ... there should be no significant difference between the number of words recalled from a list of 20 unrelated words compared to the number of categories represented by the recalled words of a list of 20 categories. (p. 227)

He found that when $T$ was held constant across lists, there was no significant difference between recall of unrelated words from List 1 and recall of categories from List L. This can be viewed as supporting the chunk hypothesis. However, when the $T$ requirement was not fulfilled, the number of categories recalled was higher than the number of unrelated words recalled.

The major question of the present article is whether the chunk hypothesis $\left[W\left(n^{\prime}\right)=C(g, z)\right]$ may apply to recognition of faces. Our attempt to answer this question led to development of the present article's conjecture.

\section{The Face Recognition by Similarity (FRBS) Conjecture and Previous Preliminary Findings}

To answer the question above, the following two crude analogies were drawn: (1) a list consisting of words and

S.S.Rakover, rakover@psy.haifa.ac.il 
categories versus a face consisting of facial values and similarity groups per facial dimension, and (2) recall of unrelated words and categories versus recognition of facial values and similarity groups. Note that one may conceive a category of related words as a semantic chunk and a group of similar features in a dimension, such as round eyes, as a similarity group, visual category, or a visual chunk. To explain this, we shall first clarify the terms relevant to face recognition and then present our answer.

A face is defined as a vector of facial values (e.g., big eyes, small mouth) where each value belongs to a different facial dimension (e.g., eyes, mouth). For example, division of a face into five dimensions $(k=5$; hair and forehead, eyes, nose, mouth, and chin) and three values per dimension $(n=3)$ generates $3^{5}=243$ different faces. One of these composites can be used in an experiment as a target face $\left(F_{\mathrm{t}}\right)$, the face to be remembered, and the rest as test faces.

Given a facial dimension, a similarity group consists of a group of facial values that are visually similar. The values in each dimension are divided into several groups in terms of their visual similarity. That is, we divide $n$ facial values per dimension into $g$ similarity groups, where the number of values per similarity group is $z=n / g$ (see Figure 1, which depicts three similarity groups of hair and forehead). One of these $g$ similarity groups is the $F_{\mathrm{t}}$ similarity group: It includes an $F_{\mathrm{t}}$ value (a value that belongs to $F_{\mathrm{t}}$ ) as one of its $z$ values. For example, in Figure 1, if $F_{\mathrm{t}}$ is bald, $g_{1}$ is the $F_{\mathrm{t}}$ similarity group.

The FRBS conjecture treats the relation between the recognition of two different target faces, $F_{\mathrm{t}}(g, z)$ and $F_{t}\left(n^{\prime}\right)$, which are associated with two experimental situations: $F_{\mathrm{t}}(g, z)$ is associated with the $G_{\mathrm{t}}(g, z)$ situation, and $F_{\mathrm{t}}\left(n^{\prime}\right)$ is associated with the $V_{\mathrm{t}}\left(n^{\prime}\right)$ situation (see below). In analogy to recall of categories from $\mathrm{L}$, the first situation, which generates $F_{\mathrm{t}}(g, z)$, treats the recognition of $F_{\mathrm{t}}(g, z)$ similarity groups. In analogy to recall of unrelated words from 1 , the second situation, which generates $F_{\mathrm{t}}\left(n^{\prime}\right)$, treats the recognition of $F_{\mathrm{t}}\left(n^{\prime}\right)$ values.

The $G_{t}(g, z)$ situation. In this situation, faces are generated on the basis of $n$ values per dimension. One of these faces is randomly selected as $F_{\mathrm{t}}(g, z)$. We divide $n$ facial values per dimension into $g$ similarity groups, where the number of values per similarity group is $z=n / g$. For example, given that Dimension A (hair and forehead) includes $n=9$ values, a division of these values into $g=3$ similarity groups appears as follows: $A_{1}\left(a_{1} a_{2} a_{3}\right), A_{2}\left(a_{4} a_{5} a_{6}\right)$, $A_{3}\left(a_{7} a_{8} a_{9}\right)$, where $A_{i}$ signifies similarity groups in Dimension $\mathrm{A}$ and $a_{j}$ signifies values. A similarity group consists of $z$ facial values that are visually similar. One of these $g$ similarity groups is the $F_{\mathrm{t}}(g, z)$ similarity group; it includes $F_{\mathrm{t}}(g, z)$ value as one of its $z$ values. The generation of the similarity groups for each dimension is made before and independently of $F_{\mathrm{t}}(g, z)$ recognition. The recognition of $F_{\mathrm{t}}(g, z)$ can be measured by two indexes: $V_{\mathrm{t}}(n)$, which counts how many $F_{\mathrm{t}}(g, z)$ values were recognized correctly, and $G_{\mathrm{t}}(g, z)$, which counts how many $F_{\mathrm{t}}(g, z)$ similarity groups were recognized correctly. The $F_{\mathrm{t}}(g, z)$ similarity group is recognized when at least one value that belongs to it is recognized (see below). In this situation, we are inter- ested in counting the number of $F_{\mathrm{t}}(g, z)$ similarity groups recognized correctly, $G_{\mathrm{t}}(g, z)$, where $G_{\mathrm{t}}(g, z)$ is dependent on $g$ and $z$, and $0 \leq G_{\mathrm{t}}(g, z) \leq 5$.

The $V_{t}\left(n^{\prime}\right)$ situation. In this situation, faces are generated on the basis of $n^{\prime}$ values per dimension. One of these faces is randomly selected as $F_{\mathrm{t}}\left(n^{\prime}\right)$. This situation is based on the foregoing situation: We randomly select for each dimension one value from each similarity group in the $G_{\mathrm{t}}(g, z)$ situation. For example, given Dimension A in the example above, in the present situation Dimension A includes three values: $a_{2} a_{4} a_{7}$, where one value was randomly sampled from $A_{1}$, one from $A_{2}$, and one from $A_{3}$ (see Figure 2). Thus, the number of values in each dimension $\left(n^{\prime}\right)$ in the present situation is equal to the number of the similarity groups that were described in the previous situation - that is, $n^{\prime}=g\left(n>n^{\prime}\right)$. Furthermore, because of this selection method, there are no similarity groups in the present situation. We calculate the number of $F_{\mathrm{t}}\left(n^{\prime}\right)$ values [values that compose $F_{\mathrm{t}}\left(n^{\prime}\right)$ ] recognized correctly, $V_{\mathrm{t}}\left(n^{\prime}\right)$, where $V_{\mathrm{t}}\left(n^{\prime}\right)$ is dependent on $n^{\prime}$, and $0 \leq V_{\mathrm{t}}\left(n^{\prime}\right) \leq 5$.

While the question of whether $W\left(n^{\prime}\right)=C(g, z)$ is answered positively, the question of whether $G_{\mathrm{t}}(g, z)=V_{\mathrm{t}}\left(n^{\prime}\right)$ is answered negatively. Accordingly, the FRBS conjecture describes the following particular relationship:

$$
G_{\mathrm{t}}(g, z) \leq V_{\mathrm{t}}\left(n^{\prime}\right),
$$

where $g=n^{\prime}, V_{\mathrm{t}}\left(n^{\prime}\right)$ depends on $n^{\prime}$ and $G_{\mathrm{t}}(g, z)$ depends on $g$ and $z$. In other words, the FRBS conjecture states that the number of $F_{\mathrm{t}}\left(n^{\prime}\right)$ values recognized correctly for $F_{\mathrm{t}}\left(n^{\prime}\right)$ in the $V_{\mathrm{t}}\left(n^{\prime}\right)$ situation is equal to or greater than the number of $F_{\mathrm{t}}(g, z)$ similarity groups recognized correctly for $F_{\mathrm{t}}(g, z)$ in the $G_{\mathrm{t}}(g, z)$ situation. (The theoretical foundations of the FRBS conjecture will be described below.)

\section{Similarity Groups Hair and Forehead}
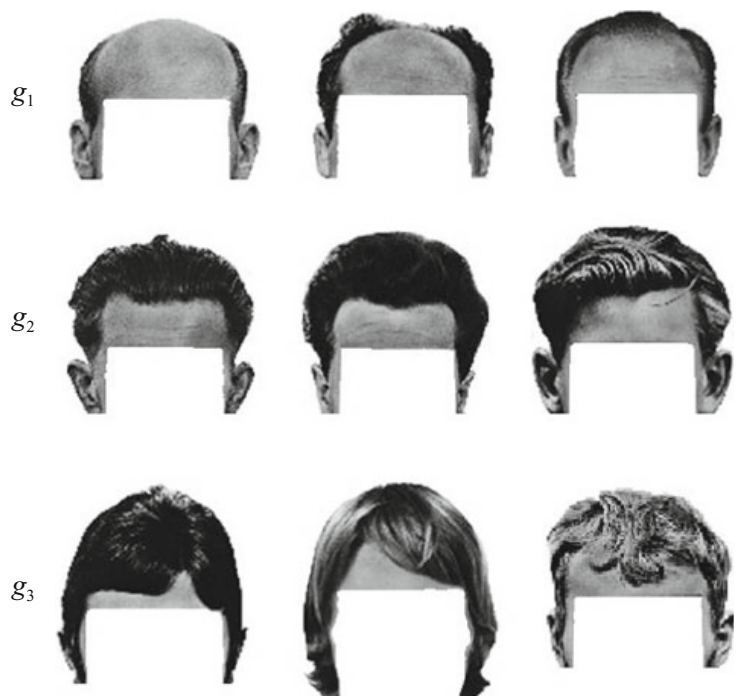

Figure 1. Three similarity groups for the hair and forehead dimension used in the present study. 


\begin{tabular}{|c|c|c|c|c|c|c|c|c|c|c|}
\hline & \multicolumn{5}{|c|}{$G_{t}(g, z)$ Situation } & \multicolumn{5}{|c|}{$V_{t}\left(n^{\prime}\right)$ Situation } \\
\hline \multirow{4}{*}{$g_{1}$} & A & B & $\mathrm{C}$ & $\mathrm{D}$ & $\mathrm{E}$ & A & B & $\mathrm{C}$ & $\mathrm{D}$ & $\mathrm{E}$ \\
\hline & $a_{1}$ & $b_{1}$ & $c_{1}$ & $d_{1}$ & $e_{1}$ & $a_{2}$ & $b_{3}$ & $c_{3}$ & $d_{1}$ & $e_{2}$ \\
\hline & $a_{2}$ & & & & $e_{2}$ & $a_{4}$ & $b_{6}$ & $c_{5}$ & $d_{5}$ & $e_{6}$ \\
\hline & $a_{3}$ & & & & $e_{3}$ & $a_{7}$ & $b_{7}$ & $c_{8}$ & $d_{9}$ & $e_{9}$ \\
\hline \multirow{3}{*}{$g_{2}$} & $a_{4}$ & & & & $e_{4}$ & \multirow{4}{*}{\multicolumn{5}{|c|}{$\begin{array}{l}\text { The values in the } V_{\mathrm{t}}\left(n^{\prime}\right) \text { situation } \\
\text { are randomly selected, one value } \\
\text { from each similarity group in the } \\
G_{\mathrm{t}}(g, z) \text { situation. Thus, } n^{\prime}=g \text {. }\end{array}$}} \\
\hline & $a_{5}$ & & & & $e_{5}$ & & & & & \\
\hline & $a_{7}$ & & & & $e_{7}$ & & & & & \\
\hline \multirow[t]{2}{*}{$g_{3}$} & $a_{8}$ & & & & $e_{8}$ & & & & & \\
\hline & $a_{9}$ & $b_{9}$ & $c_{9}$ & $d_{9}$ & $e_{9}$ & & & & & \\
\hline
\end{tabular}

Figure 2. A schematic example of the $G_{t}(g, z)$ situation and the $V_{t}\left(n^{\prime}\right)$ situation, where the values in the latter situation are randomly sampled from the former one, $g=n^{\prime}$. Capital letters signify facial dimensions, small letters signify facial values, and $g_{i}$ signifies similarity groups.

Procedures for measuring $V_{\mathrm{t}}(n), V_{\mathrm{t}}\left(n^{\prime}\right)$, and $G_{\mathrm{t}}(g, z)$. Since the customary recognition procedures were not designed for calculating how many $F_{\mathrm{t}}$ values and how many $F_{\mathrm{t}}$ similarity groups were recognized correctly, it was necessary to modify them to attain this goal. In the present study, we modified the two-alternative forced choice $(2 \mathrm{AFC})$ procedure and the yes $/$ no $(\mathrm{Y} / \mathrm{N})$ procedure (see below and the appendixes). Given this, the question that arose was whether the modified procedures lead to a correct recognition of $F_{\mathrm{t}}$, at least theoretically. If this cannot be shown, the validity of the measures is impaired. As will be seen below, Rakover and Cahlon (1989, 1999, 2001) showed mathematically that under certain conditions (called the Catch model), $F_{\mathrm{t}}$ is recognized correctly (see also the appendixes).

1. Experimental procedure. This was based on the two test faces $(2 \mathrm{TF})$ procedure developed by Rakover and Cahlon $(1989,1999)$. Briefly, the 2TF procedure is a modified $2 \mathrm{AFC}$ procedure and it was based on two stages. In the study stage, a participant was presented with $F_{\mathrm{t}}$ for $20 \mathrm{sec} . F_{\mathrm{t}}$ was randomly selected from all possible $n^{5}$ (or $n^{\prime 5}$ ) faces. In the test stage, the participant was presented with a series of 300 test pairs (randomly sampled from all possible pairs), which were presented on a computer monitor successively. Each test pair consisted of two whole test faces $(F \mathrm{~s})$. Neither of the test faces was $F_{\mathrm{t}}$. (For an illustration, see Figure 3. The faces are composites made of $k=5$ dimensions with $n=9$ values per dimension. For further explanations, see below.) The participant was required to choose from these two test faces the one that most resembled the remembered $F_{\mathrm{t}}$. The test faces were presented for $10 \mathrm{sec}$. (All the participants were psychology students, 21-23 years of age, who volunteered for the experiments for course credit.) Four or five $F_{\mathrm{t}}$ s were randomly selected for each experiment. Each $F_{\mathrm{t}}$ was associated with a different series of 300 test pairs, which were randomly selected from all possible pairs. (After the completion of an experiment, we checked for significant differences in $F_{\mathrm{t}}$ recognition among the four or five $F_{\mathrm{t}} \mathrm{s}$. No significant differences were found.)

2. Data analysis. Given the 300 choices of a participant, $F_{\mathrm{t}}$ was recognized by means of the following three-step data analysis (called the reconstruction rule by Rakover \& Cahlon, 1989, 1999, 2001).

a. For each test pair, the values that appear in the chosen test face $(F)$ but are not matched in the nonchosen $F$ are recorded. We call these the differentiating values - that is, the values that distinguish the chosen face from the nonchosen face in each pair.

b. For each facial dimension, the one differentiating value chosen most frequently by the participant in 300 choice trials (i.e., the value associated with the highest frequency of choice) is selected.

c. $F_{\mathrm{t}}$ is recognized by means of the values selected in step b.

Given the three-step data analysis and an additional similarity rule for deciding which of the two $F_{\mathrm{s}}$ is selected as most resembling the remembered $F_{\mathrm{t}}$, Rakover and Cahlon $(1989,1999,2001)$ showed mathematically that $F_{\mathrm{t}}$ is indeed recognized correctly [i.e., $V_{\mathrm{t}}(n)=5$ ]. We also found that a computer simulation-based on the threestep data analysis, the similarity rule, and $F_{\mathrm{t}}$ and its associated test pairs employed in each experiment reported here and in Rakover and Cahlon (2001) - recognized $F_{\mathrm{t}}$ in full. (Note that Rakover, 2005, developed these ideas into a special methodology for reconstruction of past events from memory.)

Briefly, the similarity rule counts, for each $F$, the number of matches - that is, the number of values common to $F_{\mathrm{t}}$ and $F$. We denote the number of matches by $\mu\left(W_{F}\right)$. The $F$ with the highest number of matches or $\mu\left(W_{F}\right)$ is selected. The similarity rule described here is characteristic of many similarity rules or similarity models reported in the literature (e.g., Estes, 1994; Medin \& Schaffer, 1978; 


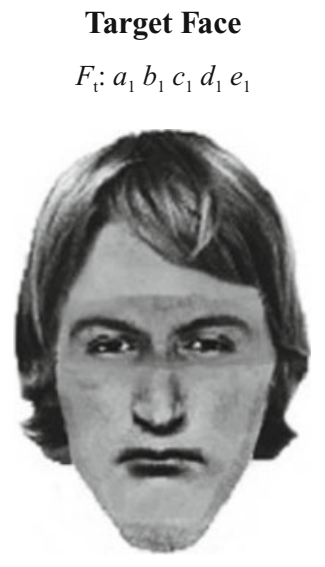

Test Faces
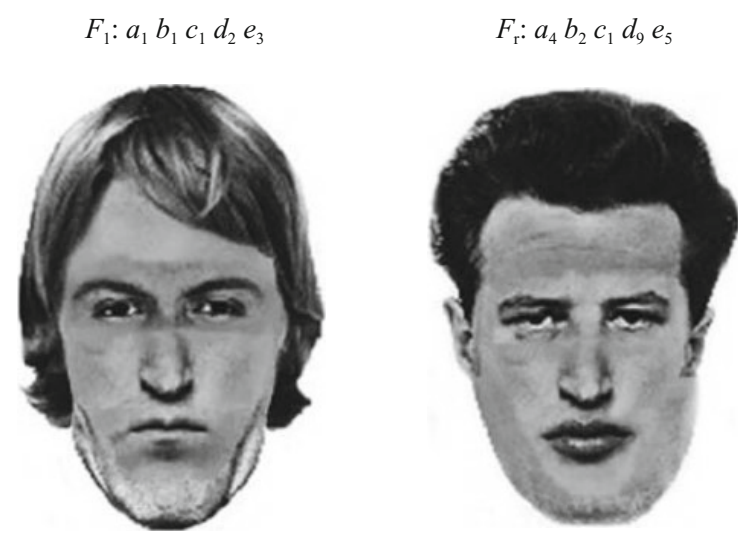

$$
\begin{gathered}
F_{1} \text { is chosen, since } \\
{\left[\mu\left(W_{F}\right)_{1}=3\right]>\left[\mu\left(W_{F}\right)_{\mathrm{r}}=1\right]}
\end{gathered}
$$

Figure 3. An example of a target face $\left(F_{t}\right)$ and a test pair: $F_{1}$ versus $F_{\mathrm{r}} . F_{1}$ is chosen, since $\left[\mu\left(W_{F}\right)_{1}=3\right]>\left[\mu\left(W_{F}\right)_{\mathrm{r}}=1\right]$.

Navarro \& Lee, 2004; Nosofsky, 1984; Tversky, 1977; for reviews and discussions, see Goldstone, 1999; Melara, 1992; Nosofsky, 1992). Among all these, the featural models, which are based on measuring similarity by considering the features that constitute an object or a stimulus, are the most relevant for the present case. As can be seen, our similarity rule can be classified as taking the featural model's approach, since it calculates the similarity between $F$ and $F_{\mathrm{t}}$ in terms of common features (facial values; see Figure 3).

We designate the number of values recognized correctly by $V_{\mathrm{t}}(n)$, where $V_{\mathrm{t}}(n)$ depends on $n\left[0 \leq V_{\mathrm{t}}(n) \leq 5\right]$. Similarly, we define $V_{\mathrm{t}}\left(n^{\prime}\right)$, where $n>\left(n^{\prime}=g\right)$. (To recapitulate: $n=g z$, where $g=n^{\prime}$.)

3. Preparation of the similarity groups. The similarity groups for each of the $k=5$ facial dimensions (hair and forehead, eyes, nose, mouth, and chin) were generated em- pirically (see Rakover \& Cahlon, 2001). Twenty-two participants were instructed to divide 9 facial values (sampled from Penry's [1971a, 1971b] Photo-fit kit) in each facial dimension into three similarity groups. Each group had to contain three similar facial values, such that their similarity was greater to each other than to the values of the other two groups (see Appendix A). An additional experiment (with 22 new participants) showed that when one of the three similarity groups was eliminated, the division of the remaining 6 values per dimension into two similarity groups preserved the previous division based on 9 values. This set of 45 facial values ( $n=5$ dimensions, with 9 values per dimension) was used in the experiments reported in Rakover and Cahlon (2001) and also in the present study.

4. Extended data analysis. We extended the above three-step data analysis for recognizing $F_{\mathrm{t}}$ similarity groups. Given the 300 choices of a participant and Facial Dimension A (forehead and hair), the computer looks first for the value with maximal frequency of choice. Then it checks whether this value belongs to the $F_{\mathrm{t}}$ similarity group (determined beforehand; see above). If it does, the computer indicates that the $F_{\mathrm{t}}$ similarity group of Facial Dimension A has been recognized. The same procedure is applied to Facial Dimensions B, C, D, and E. (For another possible extended procedure, see note 1.)

We designate the number of $F_{\mathrm{t}}$ similarity groups recognized correctly by $G_{\mathrm{t}}(g, z)$, where $G_{\mathrm{t}}(g, z)$ depends on $g, z\left[0 \leq G_{\mathrm{t}}(g, z) \leq 5\right]$.

Previous experimental support for the FRBS conjecture. The following experiment yielded preliminary empirical support for the FRBS conjecture (see Rakover $\&$ Cahlon, 2001). One hundred participants, who followed the $2 \mathrm{TF}$ procedure, were randomly assigned to four experimental groups, 25 to each group. There were four experimental groups, on the basis of which different $F_{\mathrm{t}} \mathrm{s}$ and test faces were generated.

The $\left(n^{\prime}=2\right)$ group was based on $32\left(2^{5}\right)$ faces and 466 test pairs. Each dimension consisted of two different values. For a given facial dimension, two values were chosen randomly from two different similarity groups of the $(g=2$, $z=3$ ) group, each value from a different similarity group.

The $\left(n^{\prime}=3\right)$ group was based on $243\left(3^{5}\right)$ faces and 29,403 test pairs. Each dimension consisted of three different values. For a given facial dimension, three values were chosen randomly from three different similarity groups of the ( $g=3, z=3)$ group, each value from a different similarity group.

The $(g=2, z=3)$ group was based on 7,776 (6 $\left.6^{5}\right)$ faces and 30,229,200 test pairs. Each dimension consisted of two similarity groups with three values per dimension. For a given facial dimension, two similarity groups were chosen randomly from the three similarity groups of the $(g=3, z=3)$ group.

The $(g=3, z=3)$ group was based on 59,049 ( $\left.9^{5}\right)$ faces and 1,743,362,676 test pairs. Each dimension consisted of three similarity groups with three values per dimension.

An analysis of the results, presented in Table 1, provides support for the FRBS conjecture: $G_{\mathrm{t}}(g, z) \leq V_{\mathrm{t}}\left(n^{\prime}\right)$.

1. The difference $\left[V_{\mathrm{t}}(2)=4.20\right]>\left[G_{\mathrm{t}}(2,3)=3.52\right]$ is statistically significant $[t(48)=3.83, p<.05]$. 
Table 1

Means of $V_{\mathrm{t}}\left(n^{\prime}\right)$ and of $G_{\mathrm{t}}(g, z)$ in the $V_{\mathrm{t}}\left(n^{\prime}\right)$ Situation and the $G_{t}(g, z)$ Situation

\begin{tabular}{cc}
\hline$V_{\mathrm{t}}\left(n^{\prime}\right)$ Situation & $G_{\mathrm{t}}(g, z)$ Situation \\
\hline$V_{\mathrm{t}}(2)=4.20$ & $G_{\mathrm{t}}(2,3)=3.52$ \\
$V_{\mathrm{t}}(3)=3.28$ & $G_{\mathrm{t}}(3,3)=2.88$ \\
\hline
\end{tabular}

2. The difference $\left[V_{\mathrm{t}}(3)=3.28\right]>\left[G_{\mathrm{t}}(3,3)=2.88\right]$ is not statistically significant.

Thus, the results did not show that $G_{\mathrm{t}}(g, z)$ was significantly greater than $V_{\mathrm{t}}\left(n^{\prime}\right)$, a finding that would have disconfirmed the FRBS conjecture.

Since the FRBS conjecture was established in an intuitive theoretical framework and supported by limited empirical evidence (see Rakover \& Cahlon, 2001), we decided to base it here on sound theoretical ground and broad empirical data. The empirical work includes an extension of the reported experiment and tests of the FRBS conjecture with two new experimental tasks or procedures. Hence, the present article is arranged as follows. Part 1 describes the theoretical foundations of the FRBS conjecture. Part 2 describes new experimental work on the FRBS conjecture. Part 3, a general discussion, deals with the FRBS conjecture and its relation to categorization (organization) in free recall, explanation, similarity, and interference theory.

\section{PART 1 \\ The Theoretical Basis of the FRBS Conjecture}

The reasons for the FRBS conjecture are revealed through a comparison of the $G_{t}(g, z)$ situation with the $V_{\mathrm{t}}\left(n^{\prime}\right)$ situation under two similarity conditions: when the similarity among the values in a similarity group of a given facial dimension is (1) maximal and (2) nonmaximal.

Condition 1: Maximal similarity. Since the degree of similarity between facial values in each similarity group is maximal, no differences exist among the values in a similarity group; each value is identical to the others. Therefore, each similarity group contains only one value $(z=1)$, and the number of values per dimension is $g$. Hence, under Condition 1, the $G_{\mathrm{t}}(g, z)$ situation is transformed into the $V_{\mathrm{t}}\left(n^{\prime}\right)$ situation, and therefore, $G_{\mathrm{t}}(g, z)=V_{\mathrm{t}}\left(n^{\prime}\right)$.

The interpretation that maximal similarity means identity was assumed by the geometrical models of similarity (see Goldstone, 1999; Markman, 1999; Tversky, 1977). Hahn and Chater (1998) suggested that our basic intuition states that maximal similarity is identity.

Condition 2: Nonmaximal similarity. Since the degree of similarity between facial values in each similarity group is not maximal, differences exist among the values in each similarity group. Hence, under Condition 2, each similarity group has $z$ values, $z=n / g$, and $n$ values per facial dimension, whereas in the $V_{\mathrm{t}}\left(n^{\prime}\right)$ situation there are $n^{\prime}=g$ values per dimension $\left(n>n^{\prime}\right)$. We hypothesize that the visual similarity among values impairs the recognition of $F_{\mathrm{t}}$ values as well as of $F_{\mathrm{t}}$ similarity groups. Accordingly, one tends to confuse a given value $(v)$ with another value $\left(v^{\prime}\right)$ that is visually similar to $v$. The greater the similarity between $v$ and $v^{\prime}$, the greater the probability of a similarity confusion error. We call this the similarity confusion hypothesis. Similar ideas have been supported by the results of many experiments and observations (e.g., Penry, 1971a; Shepard, 1972, 1987; Shepard \& Podgorny, 1978). Given this hypothesis, we supported the FRBS conjecture by calculating the effects of similarity confusion on $G_{\mathrm{t}}(g, z)$ and on $V_{\mathrm{t}}\left(n^{\prime}\right)$ in the following way (see Appendixes A and B).

Similarity confusion and $\boldsymbol{G}_{\mathrm{t}}(\boldsymbol{g}, \boldsymbol{z})$. Given a dimension, we assume that the values included in the non- $F_{\mathrm{t}}$ similarity groups impair the recognition of the values included in an $F_{\mathrm{t}}$ similarity group. For example, we propose that recognition of the values $\left(a_{1} a_{2} a_{3}\right)$, which are included in an $F_{\mathrm{t}}$ similarity group of Dimension A, is impaired by the values $\left(a_{4} a_{5} a_{6}\right)$ and $\left(a_{7} a_{8} a_{9}\right)$, which are included in the two non- $F_{\mathrm{t}}$ similarity groups of Dimension A.

Similarity confusion and $\boldsymbol{V}_{\mathbf{t}}\left(\boldsymbol{n}^{\prime}\right)$. Given a dimension, we assume that the non- $F_{\mathrm{t}}$ values impair recognition of an $F_{\mathrm{t}}$ value. For example, assuming that Dimension A consists of three values $\left(a_{1} a_{4} a_{7}\right)$, where $a_{1}$ is an $F_{\mathrm{t}}$ value, we propose that values $a_{4}$ and $a_{7}$ impair the recognition of $a_{1}$.

Given these assumptions, (1) we calculated, for each case, the expected value of making errors of similarity confusion - that is, the expected similarity error associated with the $G_{\mathrm{t}}(g, z)\left[E G_{\mathrm{t}}(g, z)\right]$ and with the $V_{\mathrm{t}}\left(n^{\prime}\right)$ $\left[E V_{\mathrm{t}}\left(n^{\prime}\right)\right]$ - and (2) we showed that $E G_{\mathrm{t}}(g, z)>E V_{\mathrm{t}}\left(n^{\prime}\right)$, (see Appendixes A and B).

Given the above, we proposed the FRBS conjecture: $G_{\mathrm{t}}(g, z) \leq V_{\mathrm{t}}\left(n^{\prime}\right)$, where $n^{\prime}=g$.

\section{PART 2}

\section{New Experimental Work on the FRBS Conjecture}

\section{Experiment 1 Replication and Extension}

The experiment above supported the FRBS conjecture empirically by using only some of the possible similarity groups in the $G_{\mathrm{t}}(g, z)$ situation. It did not use two groups: the $(g=2, z=2)$ group and the $(g=3, z=2)$ group. In the present experiment, we replicated and extended the experiment above by comparing (1) $V_{\mathrm{t}}\left(n^{\prime}=2\right)$ with $G_{\mathrm{t}}(g=2, z=2)$ and $G_{\mathrm{t}}(g=2, z=3)$ and $(2) V_{\mathrm{t}}\left(n^{\prime}=3\right)$ with $G_{\mathrm{t}}(g=3, z=2)$ and $G_{\mathrm{t}}(g=3, z=3)$, where $\left(n^{\prime}=g\right)=2,3$ and $z=2,3$, in a factorial experiment based on two variables: $g(2,3)$ and $z(2,3)$.

In addition to the main question of the present experiment (will the experiment empirically support the FRBS conjecture?), its factorial design allows us also to examine the effects of $g$ and $z$ on face recognition. We propose that the $G_{\mathrm{t}}(g, z)$ situation contains two sources for generating errors due to similarity confusion: One is associated with $g$ (the number of similarity groups per dimension), the other with $z$ (the number of values per similarity group). We designate the probability of errors of similarity confusion associated with $g$ by $p\left(e_{g}\right)$, where $p\left(e_{g}\right)$ increases as a function of $g$, and the probability of errors of similarity confusion associated with $z$ by $p\left(e_{z}\right)$, where $p\left(e_{z}\right)$ increases as a function of $z$. [Note that the $V_{\mathrm{t}}\left(n^{\prime}\right)$ situation contains only one source for errors of similarity confusion, $p\left(e_{g}\right)$, 
since the $V_{\mathrm{t}}\left(n^{\prime}\right)$ situation is generated from the $G_{\mathrm{t}}(g, z)$ situation by the random sampling of one value from each similarity group and, as a result, the $V_{\mathrm{t}}\left(n^{\prime}\right)$ situation does not have similarity groups.] Given this, the question is the following: For a given number of values per dimension $n$, which of the two sources of similarity confusion (one associated with $g$ and the other with $z$ ) has a more deleterious effect on the number of $F_{\mathrm{t}}$ similarity groups recognized, and which on the number of $F_{\mathrm{t}}$ values recognized?

In the case of the number of $F_{\mathrm{t}}$ similarity groups recognized, we proposed that the similarity confusion associated with $g$ would have a more deleterious effect than the similarity confusion associated with $z$ - that is, $p\left(e_{g}\right)>$ $p\left(e_{z}\right)$. In this case, the non- $F_{\mathrm{t}}$ similarity groups in a dimension are the major source of similarity confusion. Hence, we predicted that in the case in which $n=6, G_{\mathrm{t}}(g=2$, $z=3)$ would be greater than $G_{\mathrm{t}}(g=3, z=2)$, since $g$ in the former index is smaller than in the latter, so the similarity confusion will affect the latter index more than it will the former.

However, in the case of the number of $F_{\mathrm{t}}$ values recognized, we proposed that the similarity confusion associated with $z$ would have a more deleterious effect than would the similarity confusion associated with $g$ - that is, $p\left(e_{g}\right)<p\left(e_{z}\right)$. In this case, the non- $F_{\mathrm{t}}$ values within an $F_{\mathrm{t}}$ similarity group are the major source of similarity confusion. Hence, in this case, we predicted that $V_{\mathrm{t}}(g=3$, $z=2)$ would be greater than $V_{\mathrm{t}}(g=2, z=3)$, since $z$ is smaller in the former index than in the latter.

\section{Method}

One hundred twenty participants, who followed the $2 \mathrm{TF}$ procedure, were randomly assigned to six experimental groups, 20 participants per group. Five participants were assigned randomly to each of the four target faces. There were six experimental groups, on the basis of which different $F_{\mathrm{t}}$ s and test faces were generated as follows.

$(g=2, z=2)$ group. Each dimension in this group was based on two similarity groups, with two values per group. Given the ( $g=2$, $z=3$ ) group (see below), two values were chosen randomly out of three values per similarity group.

$(g=2, z=3)$ group. Each dimension in this group was based on two similarity groups, with three values per group. Given the $(g=3, z=3)$ group (see below), two similarity groups out of three similarity groups were chosen randomly.

$(g=3, z=2)$ group. Each dimension in this group was based on three similarity groups, with two values per group, which were chosen randomly from the ( $g=3, z=3)$ group, two values out of three values in a similarity group.

$(g=3, z=3)$ group. The group was based on three similarity groups, with three values per group.

$\left(n^{\prime}=2\right)$ group. Each dimension in this group was based on two values. One value was randomly sampled from each similarity group of the $(g=2, z=2)$ group.

$\left(n^{\prime}=3\right)$ group. Each dimension in this group was based on three values. One value was randomly sampled from each similarity group of the ( $g=3, z=3)$ group.

\section{Results and Discussion}

Table 2 presents the means of $V_{\mathrm{t}}\left(n^{\prime}\right)$ and of $G_{\mathrm{t}}(g, z)$ in the $V_{\mathrm{t}}\left(n^{\prime}\right)$ situation and the $G_{\mathrm{t}}(g, z)$ situation.

As can be seen from Table 2, some of the results are a good replication of the data that appear in Table 1. The results support the FRBS conjecture.
Table 2

Means of $V_{t}\left(n^{\prime}\right)$ and of $G_{t}(g, z)$ in the $V_{t}\left(n^{\prime}\right)$ Situation and the $G_{\mathrm{t}}(g, z)$ Situation

\begin{tabular}{cc}
\hline$V_{\mathrm{t}}\left(n^{\prime}\right)$ Situation & $G_{\mathrm{t}}(g, z)$ Situation \\
\hline$V_{\mathrm{t}}(2)=4.25$ & $G_{\mathrm{t}}(2,2)=3.50$ \\
& $G_{\mathrm{t}}(2,3)=3.45$ \\
$V_{\mathrm{t}}(3)=3.10$ & $G_{\mathrm{t}}(3,2)=2.55$ \\
& $G_{\mathrm{t}}(3,3)=2.95$ \\
\hline
\end{tabular}

1. The difference $\left[V_{\mathrm{t}}(2)=4.25\right]>\left[G_{\mathrm{t}}(2,2)=3.50\right]$ is statistically significant $[t(38)=2.57, p<.01]$.

2. The difference $\left[V_{\mathrm{t}}(2)=4.25\right]>\left[G_{\mathrm{t}}(2,3)=3.45\right]$ is statistically significant $[t(38)=2.83, p<.01]$.

3. The differences $\left[V_{\mathrm{t}}(3)=3.10\right]>\left[G_{\mathrm{t}}(3,2)=2.55\right]$ and $\left[V_{\mathrm{t}}(3)=3.10\right]>\left[G_{\mathrm{t}}(3,2)=2.95\right]$ are not statistically significant.

Hence, the present results did not disconfirm the FRBS conjecture by showing that $G_{\mathrm{t}}(g, z)$ is significantly greater than $V_{\mathrm{t}}\left(n^{\prime}\right)$.

Other predictions described above were partially supported. The difference $\left[G_{\mathrm{t}}(g=2, z=3)=3.45\right]>$ $\left[G_{\mathrm{t}}(g=3, z=2)=2.55\right]$ is statistically significant $[t(76)=2.83, p<.01$; the $t$ test is based on the ANOVA's $\left.M S_{\mathrm{e}}\right]$. However, the difference $\left[V_{\mathrm{t}}(g=2, z=3)=1.75\right]<$ $\left[V_{\mathrm{t}}(g=3, z=2)=2.00\right]$ is not significant.

The present experiment supports the FRBS conjecture: As in the previous experiment (see Table 1), the present results do not show that the difference $V_{\mathrm{t}}\left(n^{\prime}\right)<G_{\mathrm{t}}(g, z)$ is significant. Rather, in all cases, $V_{\mathrm{t}}\left(n^{\prime}\right)$ is greater than $G_{\mathrm{t}}(g, z)$, and in some of them this difference is statistically significant. [This raises the following question: Would a nonsignificant $G_{\mathrm{t}}(g, z)>V_{\mathrm{t}}\left(n^{\prime}\right)$ difference support the FRBS conjecture? The answer is yes, provided that we interpret empirically (as we customarily do) the relation of equality in terms of a statistically nonsignificant difference.]

Finally, whereas Rakover and Cahlon (2001) showed that recognition of $F_{\mathrm{t}}$ values and of $F_{\mathrm{t}}$ similarity groups is dependent on $n$, the present experiment indicated that for a given $n$ (where $n=g z$ ), there is a differential effect of $g$ and $z$ on face recognition.

\section{The FRBS Conjecture and \\ Two New Testing Procedures}

Can the FRBS conjecture be generalized to other experimental tasks or procedures? Would experiments with different tasks or procedures empirically support the FRBS conjecture? In the next section, we will consider two new procedures.

\section{Experiment 2 \\ The One Test Face Procedure}

In the present experiment, we employ the one test face (1TF) procedure, which is a modified version of the $\mathrm{Y} / \mathrm{N}$ recognition procedure. It is similar to the $2 \mathrm{TF}$ procedure, except for the following. Whereas in the study stage a participant is presented with $F_{\mathrm{t}}$, as in the 2TF procedure, in the test stage he or she is presented with individual test faces one at a time (in a way similar to the $\mathrm{Y} / \mathrm{N}$ recognition procedure), instead of two test faces, as in the $2 \mathrm{TF}$ 
procedure. The participant is asked to decide whether or not the test face is similar to the target face, where similarity is defined in terms of common facial values between these faces.

The difference between the $2 \mathrm{TF}$ and the $1 \mathrm{TF}$ procedures necessitates certain adaptations in the sampling method of faces and the three-step data analysis.

\section{An Adapted Sampling Method}

Since, according to the 1TF procedure, one test face is presented in the test stage, a new method has to be developed for sampling test faces from all possible test faces that will ensure that $F_{\mathrm{t}}$ values will appear in some of the test faces. (This is because the rate of faces composed of $F_{\mathrm{t}}$ values in relation to all possible faces decreases as a function of $n$. This does not occur with the 2TF procedure in which test pairs are sampled. For mathematical proofs, see Rakover \& Cahlon, 1989.) To achieve this, we use the method of sampling without replacement. The method is exemplified with $n=9$ values per dimension as follows. We randomly sampled one of the nine values of Dimension A (e.g., $a_{7}$ ); similarly, we randomly sampled one value of the nine values of Dimension B (e.g., $b_{3}$ ); and so on. The result was a test face, $F_{1}: 73581$. The next test face was created by randomly sampling one of the eight remaining values for each dimension (excluding the values that had already been sampled: 73581 ). We continued with this sampling method until the ninth test face was produced. Hence, this sampling method generated a set of nine faces. (We designated such sets of faces by $\operatorname{Set}[n], n=9$ in the present case.) Clearly, this sampling method does ensure that all $F_{\mathrm{t}}$ values will appear in some of the nine test faces. After the first Set[9] had been created, we repeated the sampling method and created $N$ more Set[9]s, totaling $9 N$ test faces. (This sampling procedure also guarantees that the frequency of appearance of each value in the sample of test faces is the same. Similarly, this guarantee is also provided by the sampling procedure for the $2 \mathrm{TF}$ procedure.)

\section{An Adapted Three-Step Data Analysis}

For each chosen $F$, all values of that $F$ are recorded. For example, if $F: 23784$ is chosen as similar to $F_{\mathrm{t}}$, the values 23784 are recorded. For each facial dimension, the value associated with the highest frequency of choice is selected as identical with the $F_{\mathrm{t}}$ value. $F_{\mathrm{t}}$ is recognized by using the five values associated with the highest frequency of choice. (Note that the extended data analysis described above can be applied directly to the present case.)

Given these adaptations, two questions arise: (1) Can these adaptations lead to $F_{\mathrm{t}}$ recognition? (2) Do these adaptations support the FRBS conjecture theoretically and empirically?

The answer to the first question is affirmative for $n>2$ (see Appendix B). Given the adapted three-step data analysis and an additional adapted similarity rule for deciding whether or not a test face $(F)$ is similar to $F_{\mathrm{t}}(F$ has to have at least one common value with $F_{\mathrm{t}}$ ), Appendix B shows mathematically that, indeed, $F_{\mathrm{t}}$ is recognized correctly.

The answer to the second question is also affirmative (see Appendix B). Since $E G(g, z)$ is greater than $E V\left(n^{\prime}\right)$ when $g=n^{\prime}$-that is, similarity confusion affects $G_{\mathrm{t}}(g, z)$ to a greater extent than it does $V_{\mathrm{t}}\left(n^{\prime}\right)$ - therefore, $V_{\mathrm{t}}\left(n^{\prime}\right) \geq$ $G_{\mathrm{t}}(g, z)$. Hence, the FRBS conjecture is supported theoretically by the $1 \mathrm{TF}$ procedure and its adaptations. The remaining question is empirical: Will the results of the present experiment support the FRBS conjecture or not?

\section{Method}

Seventy-five participants were randomly assigned to three experimental groups, 25 participants per group. In the first stage, $F_{\mathrm{t}}$ was shown, and in the second stage, 240-243 individual test faces were shown (a single $F$ each time), and the participant was asked to decide whether or not the test face was similar to the remembered $F_{\mathrm{t}}$. The generation of the similarity groups for the $G_{\mathrm{t}}(g, z)$ situation (where $g=3, z=2,3)$ and the random selection of the values from these similarity groups for the $V_{\mathrm{t}}\left(n^{\prime}\right)$ situation were based on the procedure described above. There were three experimental groups, on the basis of which different $F_{\mathrm{t}} \mathrm{s}$ and test faces were generated.

$\operatorname{ITF}(g=3, z=2)$ group. Each dimension in this group was based on three similarity groups, with two values per group; there were $40 \times \operatorname{Set}[6]=240$ test faces.

$\operatorname{ITF}(g=3, z=3)$ group. Each dimension in this group was based on three similarity groups, with three values per group; there were $27 \times \operatorname{Set}[9]=243$ test faces.

$\operatorname{ITF}\left(n^{\prime}=3\right)$ group. Each dimension in this group was based on three values per dimension. One value was randomly sampled from each of the three similarity groups of the $(g=3, z=3)$ group; there were 242 test faces $([80 \times \operatorname{Set}[3]=240]+2$ test faces, because the total possible number of faces minus $F_{\mathrm{t}}$ equals $3^{5}-1=242$ ).

As with the 2TF procedure, here too we carried out the following checks. Five $F_{\mathrm{t}} \mathrm{s}$ were randomly selected for each experimental group, and 5 participants were randomly assigned to each $F_{\mathrm{t}}$. Each $F_{\mathrm{t}}$ was associated with a different series of test faces [except for the $\operatorname{1TF}\left(n^{\prime}=3\right)$ group]. Given $F_{\mathrm{t}}$ and its associated test faces, we checked whether $F_{\mathrm{t}}$ could be recognized correctly by employing a computer simulation. The answer was yes for all $F_{\mathrm{t}}$ s. Finally, after the completion of the experiment, we checked whether there were significant differences in $F_{\mathrm{t}}$ recognition among the different $F_{\mathrm{t}} \mathrm{s}$. No significant differences were found.

\section{Results and Discussion}

Table 3 presents the means of $V_{\mathrm{t}}\left(n^{\prime}\right)$ and of $G_{\mathrm{t}}(g, z)$ in the $V_{\mathrm{t}}\left(n^{\prime}\right)$ situation and the $G_{\mathrm{t}}(g, z)$ situation.

As can be seen from Table 3, the results support the FRBS conjecture: There were nonsignificant differences between $V_{\mathrm{t}}(3)$ and $G_{\mathrm{t}}(3,2), G_{\mathrm{t}}(3,3)$. That is, the present results do not disconfirm the FRBS conjecture by showing that $G_{\mathrm{t}}(g, z)$ is significantly greater than $V_{\mathrm{t}}\left(n^{\prime}\right)$.

\section{Experiment 3 The Value Spread Procedure}

In the present experiment we employed the value spread procedure, which is a modified version of the photo spread procedure (see, e.g., Levi \& Lindsay, 2001). The value spread procedure consisted of two stages. In the study stage, a participant was presented with $F_{\mathrm{t}}$ for $20 \mathrm{sec}$; in the test stage, he or she was presented with five consecutive

Table 3

Means of $V_{t}\left(n^{\prime}\right)$ and of $G_{t}(g, z)$ in the $V_{t}\left(n^{\prime}\right)$ Situation and the $G_{\mathrm{t}}(g, z)$ Situation

\begin{tabular}{cc}
\hline$V_{\mathrm{t}}\left(n^{\prime}\right)$ Situation & $G_{\mathrm{t}}(g, z)$ Situation \\
\hline$V_{\mathrm{t}}(3)=3.40$ & $G_{\mathrm{t}}(3,2)=3.44$ \\
& $G_{\mathrm{t}}(3,3)=2.80$ \\
\hline
\end{tabular}


facial dimensions (hair and forehead, eyes, nose, mouth, and chin), where each dimension included a spread of $n$ values presented simultaneously. For example, the participant was presented with a spread of $n=9$ mouths, followed by a spread of $n=9$ noses, and so on. The participant was required to choose from each spread of $n=9$ test values the value that most resembled the remembered $F_{\mathrm{t}}$ value. A spread was presented for $10 \mathrm{sec}$. On the basis of these choices, we calculated the number of $F_{\mathrm{t}}$ values recognized $\left[V_{\mathrm{t}}(n)\right]$ and the number of $F_{\mathrm{t}}$ similarity groups recognized $\left[G_{\mathrm{t}}(g, z)\right]$. This procedure was applied to the $G_{\mathrm{t}}(g, z)$ situation and the $V_{\mathrm{t}}\left(n^{\prime}\right)$ situation.

Given the present procedure, two important questions arise. First, can we provide a mathematical proof that $F_{\mathrm{t}}$ will be successfully recognized in a way similar to those shown in the proofs offered in the previous procedures? Second, can we prove mathematically that the interference due to similarity confusion in the $G_{\mathrm{t}}(g, z)$ situation is greater than that in the $V_{\mathrm{t}}\left(n^{\prime}\right)$ situation? Although the answers to these questions are not simple, we will attempt to delineate briefly how one may go about solving them.

As to the first question (regarding the mathematical proof of $F_{\mathrm{t}}$ recognition), one way to answer this question affirmatively is to apply the similarity rule and the threestep data analysis associated with the $1 \mathrm{TF}$ and $2 \mathrm{TF}$ procedures and their appropriate mathematical proofs to the value spread procedure (see above, the appendixes, and Rakover \& Cahlon, 1989, 2001). Take, for example, the value of eyes. In the eyes spread procedure, a participant either compares the target eyes with each test eye that appears in the eyes spread and decides which test eyes resemble the target eye, or chooses from a pair of test eyes (which the participant generates from the eyes spread) the eyes most similar to the target eyes.

As to the second question [regarding the mathematical proof that $\left.E G_{\mathrm{t}}(g, z)>E V_{\mathrm{t}}\left(n^{\prime}\right)\right]$, one way to answer this question affirmatively is to apply the proofs in the two appendixes to the value spread procedure. Consider as an example the target value of eyes. As was mentioned above, we assume that a participant makes a similarity decision by comparing the remembered $F_{\mathrm{t}}$ value (eyes) with either (1) each single test value (eyes) in the eyes spread or (2) pairs of test eyes generated from the eyes spread. The application of the proofs in Appendix B to (1) shows that $\left[E G_{\mathrm{t}}(g, z)=z P(\mathrm{~m})+z(g-1)\right.$ $P(\mathrm{fa})]>\left[E V_{\mathrm{t}}\left(n^{\prime}\right)=P(\mathrm{~m})+(g-1) P(\mathrm{fa})\right]$, where $P(\mathrm{~m})$ denotes the probability of making a miss error and $P(\mathrm{fa})$ denotes the probability of making a false alarm error; the application of the proofs in Appendix B to (2) shows that $\left[E G_{\mathrm{t}}(g, z)=\right.$ $\left.(g-1) z^{2} P(\mathrm{e})\right]>\left[E V_{\mathrm{t}}\left(n^{\prime}\right)=(g-1) P(\mathrm{e})\right]$, where $P(\mathrm{e}) \mathrm{de}-$ notes the probability of making an error of similarity confusion. Hence, the similarity confusion in the $G_{\mathrm{t}}(g, z)$ situation is greater than that in the $V_{\mathrm{t}}\left(n^{\prime}\right)$ situation, and therefore, the FRBS conjecture is supported theoretically. The remaining question is empirical: Will the results of the present experiment support the FRBS conjecture or not?

\section{Method}

Sixty participants, who followed the value spread procedure, were randomly assigned to four experimental groups, 15 participants per group. For each group, five different target faces were randomly sampled, and for each target, five different value sheets were prepared; a value sheet included a random spread of $n$ facial values of a single facial dimension. Following the presentation of $F_{\mathrm{t}}$, five value sheets were presented in random order for choosing the test value most similar to the remembered $F_{\mathrm{t}}$ value. Three participants were assigned randomly to each target face. After the completion of the experiment, we checked for any significant differences in $F_{\mathrm{t}}$ recognition among the different $F_{\mathrm{t}} \mathrm{s}$. None were found. There were four experimental groups, on the basis of which different $F_{\mathrm{t}} \mathrm{s}$ and test faces were generated.

( $g=3, z=3$ ) spread group. Each dimension in this group was based on three similarity groups, with three values per group.

( $g=2, z=3$ ) spread group. Each dimension in this group was based on two similarity groups, with three values per group, where two similarity groups were chosen randomly out of three in the $(g=3, z=3)$ spread group.

$\left(n^{\prime}=3\right)$ spread group. Each dimension in this group was based on three values per dimension. One value was randomly sampled from each similarity group of the $(g=3, z=3)$ spread group.

$\left(n^{\prime}=2\right)$ spread group. Each dimension in this group was based on two values per dimension. One value was randomly sampled from each similarity group of the $(g=2, z=3)$ spread group.

\section{Results and Discussion}

Table 4 presents the means of $V_{\mathrm{t}}\left(n^{\prime}\right)$ and of $G_{\mathrm{t}}(g, z)$ in the $V_{\mathrm{t}}\left(n^{\prime}\right)$ situation and the $G_{\mathrm{t}}(g, z)$ situation.

Analysis of the results in Table 4 provides support for the FRBS conjecture: $G_{\mathrm{t}}(g, z) \leq V_{\mathrm{t}}\left(n^{\prime}\right)$.

1. The difference $\left[V_{\mathrm{t}}(2)=4.73\right]>\left[G_{\mathrm{t}}(2,3)=4.20\right]$ is statistically significant $[t(28)=2.12, p<.05]$.

2. The difference $\left[V_{\mathrm{t}}(3)=4.13\right]>\left[G_{\mathrm{t}}(3,3)=4.08\right]$ is not statistically significant.

Thus, the results did not show that $G_{\mathrm{t}}(g, z)$ is significantly greater than $V_{\mathrm{t}}\left(n^{\prime}\right)$, a finding that would have disconfirmed the FRBS conjecture.

\section{PART 3 General Discussion}

The major empirical findings of the four experiments reported here are as follows: The FRBS conjecture is supported by the $2 \mathrm{TF}$, the $1 \mathrm{TF}$, and the value spread procedures. The results did not show that $G_{\mathrm{t}}(g, z)$ is significantly greater than $V_{\mathrm{t}}\left(n^{\prime}\right)$ (a finding that would have disconfirmed the FRBS conjecture), and in all cases in which $n^{\prime}=g=2$, the difference $V_{\mathrm{t}}\left(n^{\prime}\right)>G_{\mathrm{t}}(g, z)$ was statistically significant.

Given this, we concentrate on two important topics relating to the FRBS conjecture: (1) categorization (organization) in free recall and (2) explanation, similarity, and interference theory.

\section{Categorization (Organization) in Free Recall}

Whereas Cohen's (1963) findings supported the prediction that $W\left(n^{\prime}\right)=C(g, z)$, the present findings showed that

Table 4

Means of $V_{t}\left(n^{\prime}\right)$ and of $G_{t}(g, z)$ in the $V_{t}\left(n^{\prime}\right)$ Situation and the $G_{\mathrm{t}}(g, z)$ Situation

\begin{tabular}{cc}
\hline$V_{\mathrm{t}}\left(n^{\prime}\right)$ Situation & $G_{\mathrm{t}}(g, z)$ Situation \\
\hline$V_{\mathrm{t}}(2)=4.73$ & $G_{\mathrm{t}}(2,3)=4.20$ \\
$V_{\mathrm{t}}(3)=4.13$ & $G_{\mathrm{t}}(3,3)=4.08$ \\
\hline
\end{tabular}


$W\left(n^{\prime}\right) \geq G(g, z)$. One way to explain this discrepancy is as follows. Since, in Cohen's experiments, the words in the unrelated list were different from the words in the categorized list (i.e., the former words were not sampled from the latter list), we decided to test, in a new experiment, whether $W\left(n^{\prime}\right)>C(g, z)$ when the words in the unrelated list are sampled from the categorized list.

We generated a list of $n=32$ words (nouns in Hebrew): $\mathrm{L}(g=16, z=2)$ consisted of 16 categories (such as plants, animals, furniture, food, and clothing) with 2 words per category; we then generated $1\left(n^{\prime}=16\right)$ by sampling randomly one word from each category in $\mathrm{L}(\mathrm{g}=$ $16, z=2)$. Each list was presented on a computer screen to a different group of participants (10 participants in each group). To fulfill the $T$ requirement, each word in $\mathrm{L}(g=16, z=2)$ was presented for $2 \mathrm{sec}$, whereas each word in $1\left(n^{\prime}=16\right)$ was presented for $4 \mathrm{sec}$. As in Cohen (1963) and Tulving and Pearlstone (1966) (and as in the present experiments), a category was identified when at least 1 word that belonged to it was recalled. The results show that $\left[W\left(n^{\prime}=16\right)=10.40\right]$ was not significantly different from $[C(g=16, z=2)=10.80]$.

This result confirms the chunk hypothesis. The question that arises is why, in face recognition, were there cases in which $V_{\mathrm{t}}\left(n^{\prime}\right)>G_{\mathrm{t}}(g, z)$, whereas in free recall $W\left(n^{\prime}\right)=C(g, z)$ ?

The answer probably lies in the many differences between processing facial versus verbal information. Aside from the evident differences in the experimental designs and procedures (and their associated cognitive processes; see Baddeley, 1976; Rakover \& Cahlon, 2001), we wish to emphasize the following three points.

First, although it makes sense to consider a maximum similarity among facial values in a similarity group (such as eyes), it is hard to imagine maximum similarity among words in a verbal category such as family members (mother, father, son, daughter).

Second, whereas facial values are components in an organized visual form, the face, there seems to be no such organization in a list of words. Hence, in recall of words from a list, there are no interferences equivalent to those that exist in recognition of faces: interferences of non- $F_{\mathrm{t}}$ values and non- $F_{\mathrm{t}}$ similarity groups on recognition of $F_{\mathrm{t}}$ values and $F_{\mathrm{t}}$ similarity groups, respectively.

Finally, although in our experiments the only case in which $V_{\mathrm{t}}\left(n^{\prime}\right)$ was significantly greater than $G_{\mathrm{t}}(g, z)$ was when $n^{\prime}=g=2$, it seems reasonable to assume that the number of words correctly recalled from a list containing two unrelated words $1\left(n^{\prime}=2\right)$ will be equal to the number of categories correctly recalled from a list containing two categories with two or three words per category - that is, $W\left(n^{\prime}=2\right)=C(g=2, z=2,3)$.

\section{Explanation, Similarity, and Interference Theory}

Justification of the FRBS conjecture is based on two important theoretical concepts: similarity and interference. We discriminated between two cases: when the similarity between values within a similarity group is maximal and when it is not maximal. In the maximal case, we interpreted maximal similarity as identity: If the values are maximally similar they are identical; they are duplicates. Given this, we proposed that $V_{\mathrm{t}}\left(n^{\prime}\right)=G_{\mathrm{t}}(g, z)$.

In the nonmaximal case, when the similarity among the $z$ values in a similarity group is nonmaximal, we proposed that $V_{\mathrm{t}}\left(n^{\prime}\right)>G_{\mathrm{t}}(g, z)$. The justification for this is anchored to the similarity confusion hypothesis. Given these two cases, we proposed the FRBS conjecture $V_{\mathrm{t}}\left(n^{\prime}\right) \geq G_{\mathrm{t}}(g, z)$.

Despite this explanation, the inequality suggested by this conjecture may seem, at first glance, to contradict the advantage of the configural and holistic facial information over featural information (e.g., Tanaka \& Farah, 1993; for a review, see Rakover, 2002). This contradiction is resolved if one notes that the structure of a similarity group is different from that of a face. A similarity group is a collection of visually similar values that belong to a given facial dimension. By contrast, a face is a structured, organized, visual form composed of values of different dimensions (e.g., Diamond \& Carey, 1986). Furthermore, in the present research program, we used the $n=g z$ values per dimension as the building blocks with which faces were generated and used in an experiment. Clearly, the $F_{\mathrm{t}}$ similarity group does not have the configural-holistic property that makes a face unique.

As can be seen from the above, we treated similarity as an explanatory concept, a causal one, which creates confusion among visually similar facial values. However, Sloman and Rips (1998) argued that the concept of similarity can be ranked according to its explanatory power: from a full explanatory concept, conceived of as a primitive cognitive process, to the concept endowed with no explanatory power, seen as a phenomenon to be explained, a phenomenon resulting from other, and more basic, cognitive processes.

As an explanatory concept, similarity has played a very important role in the well-known and long-standing theory called interference theory in verbal memory (e.g., Adams, 1967; Anderson, 2003; Anderson \& Bjork, 1994; Baddeley, 1976; Murdock, 1974).

Although interference theory was developed mainly for verbal material, many studies have indicated that visual similarity interferes with recognition of visual figures and faces (e.g., Baddeley, 1976; Kitagami, Sato, \& Yoshikawa, 2002; Tulving, 1981; Wells, 1988). Many researchers have found that looking at pictures of faces during the interval between learning and testing of the target face interferes retroactively with its recognition. As the number of intervening faces rises, and as their similarity to the target face increases, the effect of retroactive interference increases (for a review, see Rakover \& Cahlon, 2001). Accordingly, Rakover and Cahlon (2001) found that facial recognition decreased as a function of $n$ and $g$ and that the greater the similarity within the $F_{\mathrm{t}}$ similarity group, the smaller the $V_{\mathrm{t}}(n)$.

The similarity confusion hypothesis provides a basis for the FRBS conjecture, but it does not specify how the confusion between similar values impairs face recognition. We briefly consider here two possible hypotheses that involve encoding and retrieval processes. As $n, g$, and the similarity between facial values increase, (1) the chances of replacing $F_{\mathrm{t}}$ values with similar values and generating false $F_{\mathrm{t}}$ increase, and (2) the number of pos- 
sible composites increases, causing an increase in the difficulty of searching for $F_{\mathrm{t}}$. Rakover and Cahlon (2001) found that some experimental results tended to support Hypothesis 2 . These two hypotheses are analogous to two hypotheses proposed for explaining the misinformation effect (misinformation about an event previously seen leads people to report details that did not occur in that event). The latter two hypotheses are also based on an encoding process (misinformation distorts permanently the memory of the witnessed event) and a retrieval process (people have difficulty discriminating between the witnessed event and misinformation about it). (For thorough reviews on misinformation, see Loftus, Hoffman, \& Wagenaar, 1992; Zaragoza, Belli, \& Payment, 2007.)

In sum, the present article provides us with theoretical and empirical foundations for the FRBS conjecture. The conjecture is anchored in the theoretical approaches dealing with verbal free recall and face recognition, similarity and interference. Four experiments supported it with three different experimental procedures (tasks). Clearly, the conjecture will be reinforced if it can be generalized over different $n \mathrm{~s}, g \mathrm{~s}, z \mathrm{~s}$, experimental procedures, and stimuli.

\section{AUTHOR NOTE}

We are grateful to Morre Goldsmith and Ilan Fisher, who read the manuscript and made helpful suggestions. Thanks go to Hilla Kanner, Hen Maoz, Michal Kaufman, Miri Ben-Simon, Julia Kratshtein, Dadi Tal, and Dan Lipner, who helped run the experiments. Correspondence concerning this article should be addressed to S. S. Rakover, Department of Psychology, Haifa University, Mount Carmel, Haifa 31905, Israel (e-mail: rakover@psy.haifa.ac.il).

\section{REFERENCES}

Adams, J. A. (1967). Human memory. New York: McGraw-Hill.

Anderson, M. C. (2003). Rethinking interference theory: Executive control and the mechanisms of forgetting. Journal of Memory \& Language, 49, 415-445.

ANDERSON, M. C., \& BJork, R. A. (1994). Mechanisms of inhibition in long-term memory: A new taxonomy. In D. Dagenbach \& T. H. Carr (Eds.), Inhibitory processes in attention, memory, and language (pp. 265-326). San Diego: Academic Press.

BADDELey, A. D. (1976). The psychology of memory. New York: Basic Books.

CoHen, B. H. (1963). Recall of categorized word lists. Journal of Experimental Psychology, 66, 227-234.

DiAmond, R., \& CAREY, S. (1986). Why faces are and are not special: An effect of expertise. Journal of Experimental Psychology: General, 115, 107-117.

Estes, W. K. (1994). Classification and cognition. New York: Oxford University Press.

Goldstone, R. L. (1999). Similarity. In R. A. Wilson \& F. C. Keil (Eds.), The MIT encyclopedia of the cognitive sciences (pp. 763-764). Cambridge, MA: MIT Press

Hahn, U., \& Chater, N. (1998). Similarity and rules: Distinct? Exhaustive? Empirically distinguishable? In S. A. Sloman \& L. J. Rips (Eds.), Similarity and symbols in human thinking (pp. 111-144). Cambridge, MA: MIT Press.

Kitagami, S., Sato, W., \& YoshiKawa, S. (2002). The influence of testset similarity in verbal overshadowing. Applied Cognitive Psychology, 16, $963-972$.

LeVI, A. M., \& LindsaY, R. C. L. (2001). Lineup and photo spread procedures: Issues concerning policy recommendations. Psychology, Public Policy, \& Law, 7, 776-790.

Loftus, E. F., Hoffman, H. G., \& Wagenaar, W. A. (1992). The misinformation effect: Transformations in memory induced by postevent information. In M. L. Howe, C. J. Brainerd, \& V. F. Reyna (Eds.), Development of long-term retention (pp. 159-183). New York: Springer. Markman, A. B. (1999). Knowledge representation. Mahwah, NJ: Erlbaum.

Medin, D. L., \& Schaffer, M. M. (1978). Context theory of classification learning. Psychological Review, 85, 207-238.

Melara, R. D. (1992). The concept of perceptual similarity: From psychophysics to cognitive psychology. In D. Algom (Ed.), Psychophysical approaches to cognition (pp. 303-388). Amsterdam: North-Holland.

MiLLER, G. A. (1956). The magical number seven, plus or minus two: Some limits on our capacity for processing information. Psychological Review, 63, 81-97.

MURDOCK, B. B., JR. (1960). The immediate retention of unrelated words. Journal of Experimental Psychology, 60, 222-234.

Murdock, B. B., JR. (1974). Human memory: Theory and data. New York: Wiley.

Navarro, D. J., \& Lee, M. D. (2004). Common and distinctive features in stimulus similarity: A modified version of the contrast model. Psychonomic Bulletin \& Review, 11, 961-974.

Nosofsky, R. M. (1984). Choice, similarity, and the context theory of classification. Journal of Experimental Psychology: Learning, Memory, \& Cognition, 10, 104-114

NosofsKy, R. M. (1992). Similarity scaling and cognitive process models. Annual Review of Psychology, 43, 25-53.

PENRY, J. (1971a). Looking at faces and remembering them: A guide to facial identification. London: Elek Books.

Penry, J. (1971b). Photo-fit kit. Leeds: John Waddington of Kirkstall.

RAKOVER, S. S. (2002). Featural vs. configurational information in faces: A conceptual and empirical analysis. British Journal of Psychology, 93, 1-30.

RAKover, S. S. (2005). Two methodologies of memory research: "Explanation-testing" and "reconstruction." In J. G. Clement \& M. K. Marks (Eds.), Computer-graphic facial reconstruction (pp. 255-283). Amsterdam: Elsevier.

Rakover, S. S., \& CAHLON, B. (1989). To catch a thief with a recognition test: The model and some empirical results. Cognitive Psychology, 21, 423-468.

RaKover, S. S., \& CAHLon, B. (1999). The Catch model: A solution to the problem of saliency in facial features. Spatial Vision, 12, 73-81.

Rakover, S. S., \& CAhlon, B. (2001). Face recognition: Cognitive and computational processes. Amsterdam: John Benjamins.

SHEPARD, R. N. (1972). Psychological representation of speech sounds. In E. E. David, Jr., \& P. B. Denes (Eds.), Human communication: A unified view (pp. 67-113). New York: McGraw-Hill.

SHEPARD, R. N. (1987). Toward a universal law of generalization for psychological science. Science, 237, 1317-1323.

ShEPARD, R. N., \& Podgorny, P. (1978). Cognitive processes that resemble perceptual processes. In W. K. Estes (Ed.), Handbook oflearning and cognitive processes: Volume 5. Human information processing (pp. 189-237). Hillsdale, NJ: Erlbaum.

SlOMAN, S. A., \& RIPS, L. J. (1998). Similarity as an explanatory construct. In S. A. Sloman \& L. J. Rips (Eds.), Similarity and symbols in human thinking (pp. 1-15). Cambridge, MA: MIT Press.

TANAKA, J. W., \& FARAH, M. J. (1993). Parts and wholes in face recognition. Quarterly Journal of Experimental Psychology, 46A, 225-245.

Tulving, E. (1981). Similarity relations in recognition. Journal of Verbal Learning \& Verbal Behavior, 20, 479-496.

Tulving, E., \& Pearlstone, Z. (1966). Availability versus accessibility of information in memory of words. Journal of Verbal Learning \& Verbal Behavior, 5, 381-391.

TVersky, A. (1977). Features of similarity. Psychological Review, 84, 327-352.

Wells, G. L. (1988). Eyewitness identification: A system handbook. Toronto: Carswell.

WooD, G. (1972). Organizational processes and free recall. In E. Tulving \& W. Donaldson (Eds.), Organization of memory (pp. 49-91). New York: Academic Press.

Zaragoza, M. S., Belli, R. F., \& Payment, K. E. (2007). Misinformation effects and the suggestibility of eyewitness memory. In M. Garry \& H. Hayne (Eds.), Do justice and let the sky fall: Elizabeth Loftus and her contributions to science, law, and academic freedom (pp. 35-63). Mahwah, NJ: Erlbaum. 


\section{NOTE}

1. Is it possible to develop another rule, which will answer the question of how $F_{\mathrm{t}}$ similarity groups are recognized? The answer is yes. Given the 2TF procedure and a Facial Dimension A, the computer (1) sums the total choices (over $z$ values) in each similarity group, (2) chooses out of $g$ similarity groups the one with the highest sum total choices, and (3) checks whether this group is the $F_{\mathrm{t}}$ similarity group. If it is, the computer indicates that the $F_{\mathrm{t}}$ similarity group of Facial Dimension A has been recognized. The same procedure is applied to Facial Dimensions $\mathrm{B}, \mathrm{C}, \mathrm{D}$, and $\mathrm{E}$. This procedure is based on the hypothesis that the frequencies of choice of the values similar to the $F_{\mathrm{t}}$ value are higher than those of the values not similar to $F_{\mathrm{t}}$. In our research program, we decided to employ the extended data analysis, which is similar to the procedures employed in categorization in free recall (see the General Discussion section). Furthermore, in analyses of the $(n=9)$ group and the $(n=6)$ group reported in Rakover and Cahlon (2001, pp. 209-211), we discovered that these two procedures produced very similar $G_{\mathrm{t}}(g, z) \mathrm{s}$.

\section{APPENDIXA}

Given the $G_{\mathrm{t}}(g, z)$ situation, we shall define a similarity group when the number of facial dimensions is $k=5$, the number of facial values per dimension is $n$, the number of similarity groups is $g$, the number of values in a similarity group is $z$, and $n=g z$. Then we shall show that the expected similarity error of the $F_{\mathrm{t}}$ similarity group, denoted by $E G_{\mathrm{t}}(g, z)$, is greater than the expected similarity error of $F_{\mathrm{t}}$ values in the $V_{\mathrm{t}}\left(n^{\prime}\right)$ situation, denoted by $E V_{\mathrm{t}}\left(n^{\prime}\right)$, where $g=n^{\prime}$.

Throughout this Appendix (as well as in Appendix B), we will consider only the first facial dimension, denoted by A, but the results are the same for the second facial dimension (B), the third (C), the fourth (D), and the fifth (E). The two reasons for this approach are as follows (see Rakover \& Cahlon, 1989). First, we assume independence among facial dimensions, as well as among values, because of symmetry among these components. Hence, the model assigns the same weight to each dimension and the same weight to each value. (A solution for the case in which dimensions are assigned different weights is presented in Rakover \& Cahlon, 1999.) The second reason is mathematical simplicity: To assume certain complex dimensions and value interactions, which currently are not established theoretically and empirically, would place an unnecessary burden on the development of the present work. In contrast, adopting such a simple approach may open new fruitful directions of research.

We consider the set of all faces:

$$
\left\{\left(a_{i}, b_{j}, c_{k}, d_{t}, e_{s}\right), i=1,2, \ldots, n ; j=1,2, \ldots, n ; k=1,2, \ldots, n ; t=1,2, \ldots, n ; s=1,2, \ldots, n\right\},
$$

with the target face $F_{\mathrm{t}}=\left(a_{1}, b_{1}, c_{1}, d_{1}, e_{1}\right)$ or $F_{\mathrm{t}}=(1,1,1,1,1)$.

As was mentioned above, we focus on the first dimension, A. We divide the set of all faces into $g$ similarity groups. Each group contains $z$ values so that $z=n / g$ or $n=g z$. In this case, we have a group of target faces:

$$
G_{\mathrm{t}}=\left\{\begin{array}{l}
\left(a_{1}, b_{i}, c_{j}, d_{k}, e_{l}\right) \\
\left(a_{2}, b_{i}, c_{j}, d_{k}, e_{l}\right), \\
\vdots \\
\left(a_{z}, b_{i}, c_{j}, d_{k}, e_{l}\right)
\end{array}\right.
$$

where $i=1, \ldots, n, j=1, \ldots, n, k=1, \ldots, n, l=1, \ldots, n$.

Here, the range of indexes means the collection of faces included in the set $G_{\mathrm{t}}$. This set consists of $z^{5}-1$ faces (excluding $F_{\mathrm{t}}$ ), where each facial dimension consists of $z$ values.

\section{Similarity Groups}

We divide the set of all faces into similarity groups, the set

$$
\begin{aligned}
g_{1}=\left\{\left(a_{1}, b_{i}, c_{j}, d_{k}, e_{l}\right),\left(a_{2}, b_{i}, c_{j}, d_{k}, e_{l}\right), \ldots,\left(\mathrm{a}_{z}, \ldots\right)\right\} \text { where }\{i, j, k, l=1,2, \ldots, n\} \\
\text { and }\left(a_{1}, b_{1}, c_{1}, d_{1}, e_{1}\right) \notin g_{1} . \\
g_{2}=\left\{\left(a_{z+1}, \ldots\right),\left(a_{z+2}, \ldots,\right), \ldots,\left(a_{2 z}, \ldots,\right)\right\} \\
g_{3}=\left\{\left(a_{2 z+1}, \ldots,\right),\left(a_{2 z+2}, \ldots,\right), \ldots,\left(a_{3 z}, \ldots,\right)\right\} \\
\vdots \\
\quad: \\
g_{g}=\left\{\left(a_{g z-z+1}, \ldots,\right),\left(a_{g z-z+2}, \ldots,\right), \ldots,\left(a_{g z}, \ldots,\right)\right\}
\end{aligned}
$$

In each similarity group, the other four dimensions are B, C, D, and E with the appropriate indexes; for example, in $g_{3}$, there are $\left(a_{i}, b_{j}, c_{k}, d_{s}, e_{t}\right)$ where $i=2 z+1,2 z+2, \ldots, 3 z$ and $s, j, k, t=1, \ldots, n$.

The similarity between any two faces is a number between zero and one, denoted by $S$, relative to the first facial dimension. 


\section{APPENDIX A (Continued)}

We denote the matrix $S\left[g_{i}, g_{i}\right]=\left\{S_{j, k}\left[g_{i}, g_{i}\right] ; j=1,2, \ldots, z ; k=1,2, \ldots, z\right\}$ the similarity between faces in the group $g_{i}, i=1,2, \ldots, g$. The values $S_{j, k}\left[g_{i}, g_{i}\right]$ are the similarities between the faces in the group $g_{i}$, containing $\left(a_{(i-1) z+j}, \ldots,\right),\left(a_{(i-1) z+k}, \ldots\right)$, where the remaining dimensions have the same weights. It is obvious that

$$
0<S_{j, k}\left[g_{i}, g_{i}\right] \leq 1 ; j=1, \ldots, z, k=1,2, \ldots, z \text { and } S_{j, j}\left[g_{i}, g_{i}\right]=1 .
$$

We also define similarity between two different groups:

$S_{e, t}\left[g_{i}, g_{s}\right]=\left\{\right.$ similarity between two faces containing $\left(a_{(i-1) z+1}, \ldots,\right)$ and $\left(a_{(s-1) z+t}, \ldots,\right)$, the first in $g_{i}$ and the second in $\left.g_{s}\right\}$, and

$S_{e, t}\left[g_{i}, g_{s}\right]_{i \neq s}<S_{j, k}\left[g_{m}, g_{m}\right]$, where $e, t=1,2, \ldots, z, j, k=1,2, \ldots, z$, and $i=1,2, \ldots, g, m=1,2, \ldots$, $g, s=1,2, \ldots, g$.

Note that $S>0$ since there is always some similarity between any two faces.

Lemma 1. $E G_{t}(g, z)=\left[\left(z^{4}-1\right)+z^{4}(z-1)\left(z^{4}(n-z)\right)+16 z^{7}(n-z)^{3}+36 z^{5}(n-z)^{5}+16 z^{3}(n-z)^{7}+\right.$ $\left.(n-z)^{9} z\right] P(\mathrm{e})$, where $P(\mathrm{e})$ designates the probability of a similarity confusion error.

Proof. We consider all the faces in $g_{1}$ where $F_{\mathrm{L}}$ is taken from $g_{1}$ and $F_{\mathrm{R}}$ is taken from $g_{2}, \ldots, g_{g}$. With respect to the first dimension, we assume that $a_{1}, \ldots, a_{z}$ are values of the target face. We examine the pair $F_{\mathrm{L}}=$ $(\bullet, \mathrm{B}, \mathrm{C}, \mathrm{D}, \mathrm{E}), F_{\mathrm{R}}=(\bullet, \mathrm{B}, \mathrm{C}, \mathrm{D}, \mathrm{E})$, where $(\bullet, \mathrm{B}, \mathrm{C}, \mathrm{D}, \mathrm{E})$ in both cases have the same weight. The weight can be $4,3,2,1$, and 0 (i.e., number of matches between a test face and $F_{\mathrm{t}}$ ). Let $N_{4}$ denote all pairs with weight $4, N_{3}$ with weight $3, N_{2}$ with weight $2, N_{1}$ with weight 1 , and $N_{0}$ with weight 0 . Given this, the calculations yielded:

$$
\begin{gathered}
N_{4}^{2} z(n-z)=\left[z^{4}-1+z^{4}(z-1)\right]\left[z^{4}(n-z)\right] ; N_{3}^{2} z(n-z)=16 z^{7}(n-z)^{3} ; N_{2}^{2} z(n-z)=36 z^{5}(n-z)^{5} ; \\
N_{1}^{2} z(n-z)=16 z^{3}(n-z)^{7} ; \text { and } N_{0}^{2} z(n-z)=(n-z)^{9} z .
\end{gathered}
$$

In $N_{0}$ each facial dimension B,C,D,E contains $n-z$ values and, thus, $(\bullet, \mathrm{B}, \mathrm{C}, \mathrm{D}, \mathrm{E})$ contains $(n-z)^{4}$ and $N_{0}{ }^{2}=$ $(n-z)^{8}$; we multiply by $z(n-z)$ and obtain $(n-z)^{9} z$, since the first dimension of $F_{\mathrm{L}}$ contains $n-z$ values and $F_{\mathrm{R}}$ contains $z$ value. We calculated the remaining terms similarly.

Therefore,

$$
\begin{aligned}
E G_{\mathrm{t}}(g, z)=[ & \left(z^{4}-1+z^{4}(z-1)\right)\left(z^{4}(n-z)\right)+16 z^{7}(n-z)^{3} \\
& \left.+36 z^{5}(n-z)^{5}+16 z^{3}(n-z)^{7}+(n-z)^{9} z\right] P(\mathrm{e}),
\end{aligned}
$$

which is the result of Lemma 1.

Lemma 2. $E V_{\mathrm{t}}\left(n^{\prime}\right)=\left[16+36(g-1)^{2}+16(g-1)^{4}+(g-1)^{6}\right](g-1)^{3} P(\mathrm{e})$.

Proof. The $n^{\prime}$ values in the $V_{\mathrm{t}}\left(n^{\prime}\right)$ situation are generated from the $n$ values in the $G_{\mathrm{t}}(g, z)$ situation, $n^{\prime}=g$, by a process of random sampling. We take one value from Dimension A, Group $g_{1}$, denoted $a_{g 1}$; we do the same for $g_{2}$, and we get $a_{g_{2}}$ up to $a_{g_{g}}$. From the second dimension, B, we sample $b_{g_{1}}, \ldots, b_{g_{g}}$; from the third dimension, C, $c_{g_{1}}, \ldots, c_{g_{g}}$; from the fourth dimension, $\mathrm{D}, d_{g_{1}}, \ldots, d_{g_{g}}$; and from the fifth dimension, $\mathrm{E}, e_{g_{1}}, \ldots, e_{g_{g}}$.

For simplification, we drop the symbol $g$, so we have the table

$$
\begin{array}{lllll}
\mathrm{A} & \mathrm{B} & \mathrm{C} & \mathrm{D} & \mathrm{E} \\
a_{1} & b_{1} & c_{1} & d_{1} & e_{1} \\
: & : & : & : & : \\
: & : & : & : & : \\
a_{g} & b_{g} & c_{g} & d_{g} & e_{g}
\end{array}
$$

We have $g^{5}$ faces minus the target face, $F_{\mathrm{t}}=\left(a_{1}, b_{1}, c_{1}, d_{1}, e_{1}\right)$. To find $E V_{\mathrm{t}}\left(n^{\prime}\right)$, we take pairs $F_{\mathrm{L}}=\left(a_{1} \mathrm{~B} \mathrm{C} \mathrm{D} \mathrm{E}\right)$ and $F_{\mathrm{R}}=\left(a_{j}, \mathrm{~B} \mathrm{C} \mathrm{D} \mathrm{E}\right)$, where $j=2, \ldots, g$. As before, we assume that B C D E in $F_{\mathrm{L}}$ and in $F_{\mathrm{R}}$ have the same weight. We denote by $N_{3}$ the number of pairs $F_{\mathrm{L}}$ and $F_{\mathrm{R}}$ with weight 3 , and $N_{2}$ with weight $2, N_{1}$ with weight 1 , $N_{0}$ with weight 0 . The calculations (which are similar to those made in Lemma 1) yielded:

$$
N_{3}^{2}(g-1)=16(g-1)^{3} ; N_{2}^{2}(g-1)=36(g-1)^{5} ; N_{1}^{2}(g-1)=16(g-1)^{7} ; N_{0}^{2}(g-1)=(g-1)^{9} .
$$

Therefore,

$$
E V_{\mathrm{t}}\left(n^{\prime}\right)=\left[(g-1)^{9}+16(g-1)^{7}+36(g-1)^{5}+16(g-1)^{3}\right] P(\mathrm{e}),
$$

which is the result of Lemma 2. 


\section{APPENDIX A (Continued)}

Lemma 3. For any $n, z$, and $g$ where $g=n / z, z \geq 2$, the following inequality holds:

$$
E G_{\mathrm{t}}(g, z)>E V_{\mathrm{t}}\left(n^{\prime}\right) \text {. }
$$

Proof. Given $E G_{\mathrm{t}}(g, z)$ and $E V_{\mathrm{t}}\left(n^{\prime}\right)$ from Lemmas 1 and 2, we have that

$$
\begin{aligned}
E V_{\mathrm{t}}\left(n^{\prime}\right)= & {\left[16+36 \frac{(n-z)^{2}}{z^{2}}+16 \frac{(n-z)^{4}}{z^{4}}+\frac{(n-z)^{6}}{z^{6}}\right]\left[\frac{(n-z)^{3}}{z^{3}}\right] P(e) } \\
= & {\left[\frac{16}{z^{3}}(n-z)^{3}+\frac{36}{z^{3}}(n-z)^{5}+\frac{16(n-z)^{7}}{z^{7}}+\frac{(n-z)^{9}}{z^{9}}\right] P(e) } \\
& <\left[16(n-z)^{3}+36(n-z)^{5}+16(n-z)^{7}+(n-z)^{9}\right] P(e) \\
& <E G_{\mathrm{t}}(g, z) .
\end{aligned}
$$

Thus, $E V_{\mathrm{t}}\left(n^{\prime}\right)<E G_{\mathrm{t}}(g, z)$.

\section{APPENDIX B}

In the first part of this appendix, we will show that the 1TF procedure leads to the correct recognition of $F_{\mathrm{t}}$. In the second part, we will show that $E G_{\mathrm{t}}(g, z)>E V_{\mathrm{t}}\left(n^{\prime}\right)$.

\section{Part 1}

Theorem. Given the $1 T F$ procedure, $k=5$, and $n$ facial values per facial dimension, $F_{\mathrm{t}}$ is correctly recognized (i.e., $\mu(t)>\mu(n t)$ : see below).

Proof. In this appendix, we use the concepts and the notations we used in Rakover and Cahlon (1989) and in Appendix A. Assuming that $F_{\mathrm{t}}: a_{1} b_{1} c_{1} d_{1} e_{1}$, simple calculations show that:

$$
\mu(t)=n^{4}-1,
$$

the number of faces that start with $a_{1}$ (excluding $F_{\mathrm{t}}$,

$$
\mu(n t)=n^{4}-(n-1)^{4},
$$

the number of faces that start with $a_{2}$ (a non- $F_{\mathrm{t}}$ value that is arbitrarily chosen from all non- $F_{\mathrm{t}}$ values) and that have at least one $F_{\mathrm{t}}$ value in the remaining dimensions.

Hence, since $\mu(t)-\mu(n t)=(n-1)^{4}-1>0$ for $n>2, F_{\mathrm{t}}$ is recognized correctly.

\section{Part 2}

Lemma 1. $E G_{\mathrm{t}}(g, z)=(n-z)^{4} z P(\mathrm{~m})+(n-z)(n-z)^{4} P(\mathrm{fa})$, where $P(\mathrm{~m})$ denotes the probability of making a miss error and $P(\mathrm{fa})$ denotes the probability of making a false alarm error.

Proof. We divide the set of all faces into $n$ columns, where column 1 contains all faces starting with $a_{1}$, column 2 with $a_{2}$, and so on. The last column starts with $a_{n}$.

To find the coefficient of $P(\mathrm{~m})$, we consider all the faces in column 1 that do not contain $a_{1}, a_{2}, \ldots, a_{z}$ : There are $(n-z)^{4}$. There are $z$ columns so we have a coefficient $(n-z)^{4} z P(\mathrm{~m})$.

To find the coefficient of $P(\mathrm{fa})$, we consider the last $(n-z)$ columns. In each column, there are $(n-z)^{4}$ faces, so the coefficient of $P(\mathrm{fa})$ is $(n-z)^{4}(n-z) P(\mathrm{fa})$.

Therefore, $E G_{\mathrm{t}}(g, z)=(n-z)^{4} z P(\mathrm{~m})+(n-z)^{4}(n-z) P(\mathrm{fa})$.

Lemma 2. $E V_{\mathrm{t}}\left(n^{\prime}\right)=(g-1)^{4} P(\mathrm{~m})+(g-1)^{5} P(\mathrm{fa})$

Proof. As in Appendix A, we create the following table:

$$
\begin{array}{lllll}
\mathrm{A} & \mathrm{B} & \mathrm{C} & \mathrm{D} & \mathrm{E} \\
a_{1} & b_{1} & c_{1} & d_{1} & e_{1} \\
: & : & : & : & : \\
: & \vdots & : & : & : \\
a_{g} & b_{g} & c_{g} & d_{g} & e_{g}
\end{array}
$$

To find the coefficient of $P(\mathrm{~m})$, we consider all the faces in columns such that B C D E dimensions do not contain $a_{1}$. There are $(g-1)^{4}$ faces. 


\section{APPENDIX B (Continued)}

To find the coefficient of $P(\mathrm{fa})$, we consider the remaining columns: second, third, . ., gth column. Each column contains $(g-1)^{4}$ faces, so the coefficient of $P(\mathrm{fa})$ is $(g-1)^{4} P(\mathrm{fa})(g-1)=(g-1)^{5} P(\mathrm{fa})$.

Combining these two cases, we obtain

$$
E V_{\mathrm{t}}\left(n^{\prime}\right)=(g-1)^{4} P(\mathrm{~m})+(g-1)^{5} P(\mathrm{fa}) .
$$

Lemma 3. $E G_{\mathrm{t}}(g, z)>E V_{\mathrm{t}}\left(n^{\prime}\right)$.

Proof. Given that $z \geq 2$ and $g=n / z$,

$$
\begin{aligned}
E V_{\mathrm{t}}\left(n^{\prime}\right)= & \left(\frac{n}{z}-1\right)^{4} P(\mathrm{~m})+\left(\frac{n}{z}-1\right)^{5} P(\mathrm{fa}) \\
& <(n-z)^{4} P(\mathrm{~m})+(n-z)^{5} P(\mathrm{fa}) \\
& <(n-z)^{4} z P(\mathrm{~m})+(n-z)^{5} P(\mathrm{fa})=E G_{\mathrm{t}}(g, z) .
\end{aligned}
$$

Thus, $E V_{\mathrm{t}}\left(n^{\prime}\right)<E G_{\mathrm{t}}(g, z)$.

(Manuscript received November 16, 2007;

revision accepted for publication February 26, 2008.) 